\title{
Determining the mechanical constitutive properties of metals as a function of strain rate and temperature: A combined experimental and modeling approach
}

\section{Progress report for 2004}

Development and validation of constitutive models for polycrystalline materials subjected to high strain rate loading over a range of temperatures are needed to predict the response of engineering materials to in-service type conditions (foreign object damage, high-strain rate forging, high-speed sheet forming, deformation behavior during forming, response to extreme conditions, etc.). To account accurately for the complex effects that can occur during extreme and variable loading conditions, requires significant and detailed computational and modeling efforts. These efforts must be closely coupled with precise and targeted experimental measurements that not only verify the predictions of the models, but also provide input about the fundamental processes responsible for the macroscopic response. Achieving this coupling between modeling and experimentation is the guiding principle of this program. Specifically, this program seeks to bridge the length scale between discrete dislocation interactions with grain boundaries and continuum models for polycrystalline plasticity. Achieving this goal requires incorporating these complex dislocation-interface interactions into the well-defined behavior of single crystals. Despite the widespread study of metal plasticity, this aspect is not well understood for simple loading conditions, let alone extreme ones.

Our experimental approach includes determining the high-strain rate response as a function of strain and temperature with post-mortem characterization of the microstructure, quasi-static testing of predeformed material, and direct observation of the dislocation behavior during reloading by using the in situ transmission electron microscope deformation technique. These experiments will provide the basis for development and validation of physically-based constitutive models, which will include dislocation-grain boundary interactions for polycrystalline systems. One aspect of the program will involve the direct observation of specific mechanisms of micro-plasticity, as these will indicate the boundary value problem that should be addressed. This focus on the pre-yield region in the quasi-static effort (the elasto-plastic transition) is also a tractable one from an experimental and modeling viewpoint. In addition, our approach will minimize the need to fit model parameters to experimental data to obtain convergence. These are critical steps to reach the primary objective of simulating and modeling material performance under extreme loading conditions.

To achieve these goals required assembling a multidisciplinary team, see Table 1, with key collaborators at the National Laboratories. One of the major issues for the team members was to learn about the expertise available and how to communicate across disciplines. The communication issue is a challenging one and is being addressed in part with weekly meetings in which the graduate students present lectures on the fundamentals of their respective areas to the entire group. Breakthroughs in science are presented but these, by necessity, assume a tutorial nature; examples of student led meetings can be found at our website http://hrdg.mse.uiuc.edu/. For example, interpreting electron micrographs and understanding what can be achieved by using electron microscopy is challenging for the modeling expert as is comprehending the input and limitations of crystal plasticity codes for an electron microscopist. Significant progress has been made at dissolving these barriers and the students are able to work across the disciplines. 
Table 1. Project participants, Affiliations and Percentage of time supported by this grant.

\begin{tabular}{|c|c|c|}
\hline Principal Investigators & Department Affiliation & $\begin{array}{l}\text { Fraction of time support } \\
\text { grant }\end{array}$ \\
\hline Professor I. Robertson, & $\begin{array}{l}\text { Materials Science } \\
\text { Enqineering (MatSE) }\end{array}$ & 0.5 month \\
\hline Professor A. Beaudoin & $\begin{array}{l}\text { Mechanical and Industrial } \\
\text { Engineering (MIE) }\end{array}$ & 1 month \\
\hline Professor John Lambros & $\begin{array}{ll}\text { Aeronautical Engineering } \\
\text { (AAE) }\end{array}$ & 1 month \\
\hline \multicolumn{3}{|c|}{ (AAE) Graduate Students } \\
\hline Cindy Smith & MSE & $100 \%$ \\
\hline Bryan Miller & MSE & $100 \%$ \\
\hline Jamie Kimberley & $A E$ & \\
\hline Henry Padilla & MIE & $100 \%$ \\
\hline Satya Varadhan & MIE* & $100 \%$ \\
\hline \multirow{2}{*}{\multicolumn{3}{|c|}{$\begin{array}{l}\text { Khaled Al-Fadhalah * } \\
\text { * Supported through a matching contribution from the ASCI Level } 1 \text { Center at UIUC. } \\
\text { * Graduated }\end{array}$}} \\
\hline & & \\
\hline \multicolumn{3}{|c|}{ Collaborators at National Laboratories } \\
\hline David Lassila & Lawrence Livern & nore National Laboratory \\
\hline Vassily Bulatov & Lawrence Livern & nore National Laboratory \\
\hline Geoff Campbell & Lawrence Livern & nore National Laboratory \\
\hline Carlos Tomé & Los Alamos Nati & ional Laboratory \\
\hline Amit Misra & Los Alamos Nati & ional Laboratory \\
\hline George Kaschner & Los Alamos Nat & ional Laboratory \\
\hline Dave Korzekwa & Los Alamos Nati & ional Laboratory \\
\hline
\end{tabular}

The focus of our program has been the characterization of the competition between slip and twinning deformation modes in hep zirconium and fcc copper with an emphasis on evaluating the hardening effect of twins. There are three aspects to the program: bulk mechanical property assessment, characterization, and modeling. In each aspect multiple approaches are employed. The mechanical behavior is being probed using quasi-static compression tests on a servo-hydraulic load frame, interrupted strain dynamic compression tests via the split-Hopkinson pressure bar (SHPB), nanoindentation, and a newly developed laser-loading technique. The Hopkinson bar data provide a basis for interpretation of the results of this last technique. Characterization of the deformation structure utilizes several techniques to probe the evolution of texture and deformation mode. The interrupted strain tests provide snapshots in the strain history of the evolving microstructure and serve as the starting material for active straining experiments in situ in the transmission electron microscope which allows the evolution of the microstructure to be directly observed as a function of strain. In this report we demonstrate that deformation twins are generated by the high strain rate laser-loading technique and that initial characterization studies have revealed some unexpected observations. In the next fiscal period we will employ an in-situ thermal measurement technique, which uses a high speed infrared detector array to provide real-time, high-rate temperature measurements during SHPB tests. This will provide data to study energy stored in structures through the creation of boundaries. These experimental efforts will provide the basis for the modeling effort, which will include the impact of grain and twin boundaries in the overall response. The status of the work to date is given in the following summary. 
Two types of zirconium were tested in compression: zirconium bar, with a purity of $99 \%$ (no chemical analysis done), and a commercial purity zirconium plate (results of chemical analysis performed at UIUC shown in Table 2), cross rolled to a thickness of $25.4 \mathrm{~mm}$.

Table 2 Chemical analysis for CP Zr 702 plate material.

\begin{tabular}{|c|c|c|c|c|c|c|c|}
\hline Element & $\mathrm{Zr}$ & $\mathrm{Hf}$ & $\mathrm{Al}$ & $\mathrm{Cr}$ & $\mathrm{Fe}$ & $\mathrm{Ti}$ & $\mathrm{V}$ \\
\hline Wt \% & 97.05 & 0.50 & 0.72 & 0.02 & 0.07 & 0.02 & 0.83 \\
\hline
\end{tabular}

The zirconium bar samples were machined so that the compression axis was coincident with the bar axis and subsequently annealed at a temperature of $1100^{\circ} \mathrm{C}$ for 24 hours to achieve a large grain size. Zirconium plate samples were machined with the compression axis coincident with the through thickness direction of the plate. The starting microstructure and starting textures of these two materials are shown in Figures 1 and 2, respectively. The annealed bar has a grain size on the order of hundreds of microns, while the as-received plate has a grain size of approximately 25 microns.
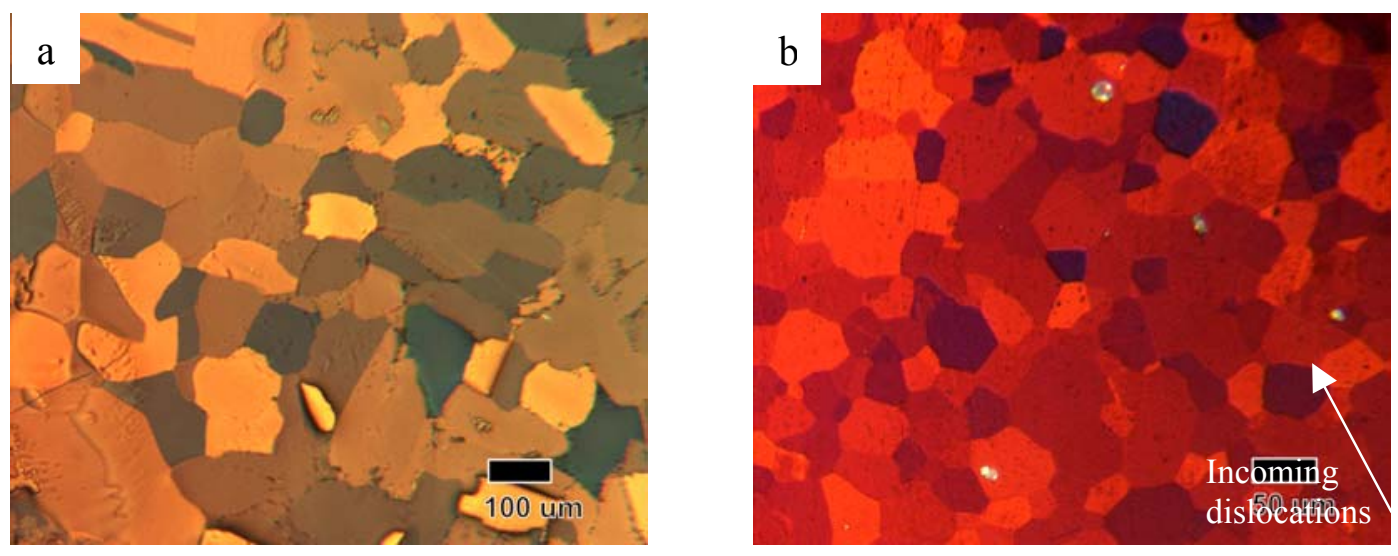

Figure 1 Starting microstructure of the (a) zirconium bar and (b) zirconium plate.

The starting texture of the annealed bar is markedly different from that of the plate. Figure 2 demonstrates that the bar has a weak overall texture, while the plate has a strong basal texture with nearly all basal poles aligned with the through thickness (compression) direction. Optical characterization of the SHPB high-rate deformed microstructures of the zirconium bar (Fig. 3a) indicates that at the strain rates tested (1200/s - 4500/s) deformation twinning occurs as a result of the high compressive stresses. Examination of the deformed microstructure in the TEM reveals twins coexisting with dislocations in

a

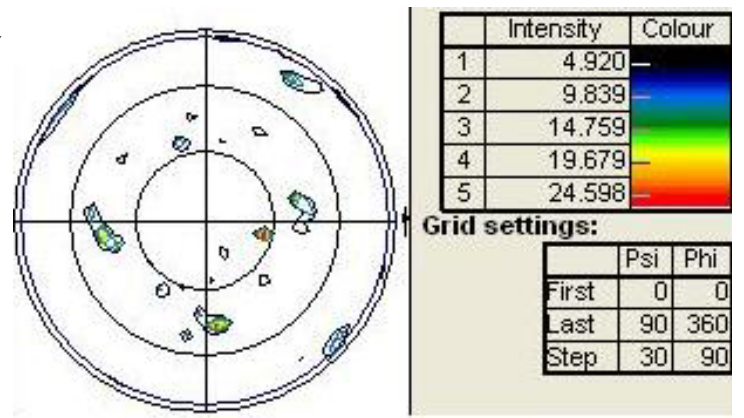

$\mathrm{b}$

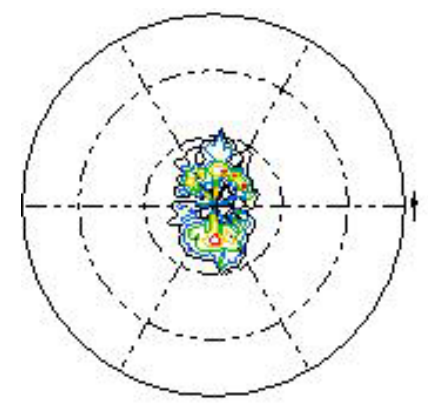

Figure 2 Starting textures of the (a) zirconium bar and (b) zirconium plate. 
some grains, Fig. 3b. Consistent with the optical micrograph twins are not present in all grains. For example, the grain shown in Fig. 3c contains only dislocations. Figure 3d shows a twin boundary being impacted by an array of dislocations and the transmission of slip across the twin boundary. Cases such as these are being analyzed to determine if the slip transmission criteria developed for FCC metals are applicable to twin boundaries in HCP materials. Within the twin several thin straight bands (marked by an arrowhead) can be seen extending from one boundary to the other. These are most probably deformation twins, created be the propagation across the twin by partial dislocations emitted from one boundary. Zhu et al. ${ }^{1}$ have recently shown that a high stress is required to initiate the emission of the first partial dislocation but a lower stress is needed for the emission of the other partial dislocations that are required to form the twin. The stress to emit the partial dislocation needed to annihilate the twin is significantly higher than the stress needed to emit the first partial dislocation. It is therefore possible to generate a stable deformation twin. This is consistent with our observations in silver in which deformation twins once nucleated propagate rapidly through the grain.
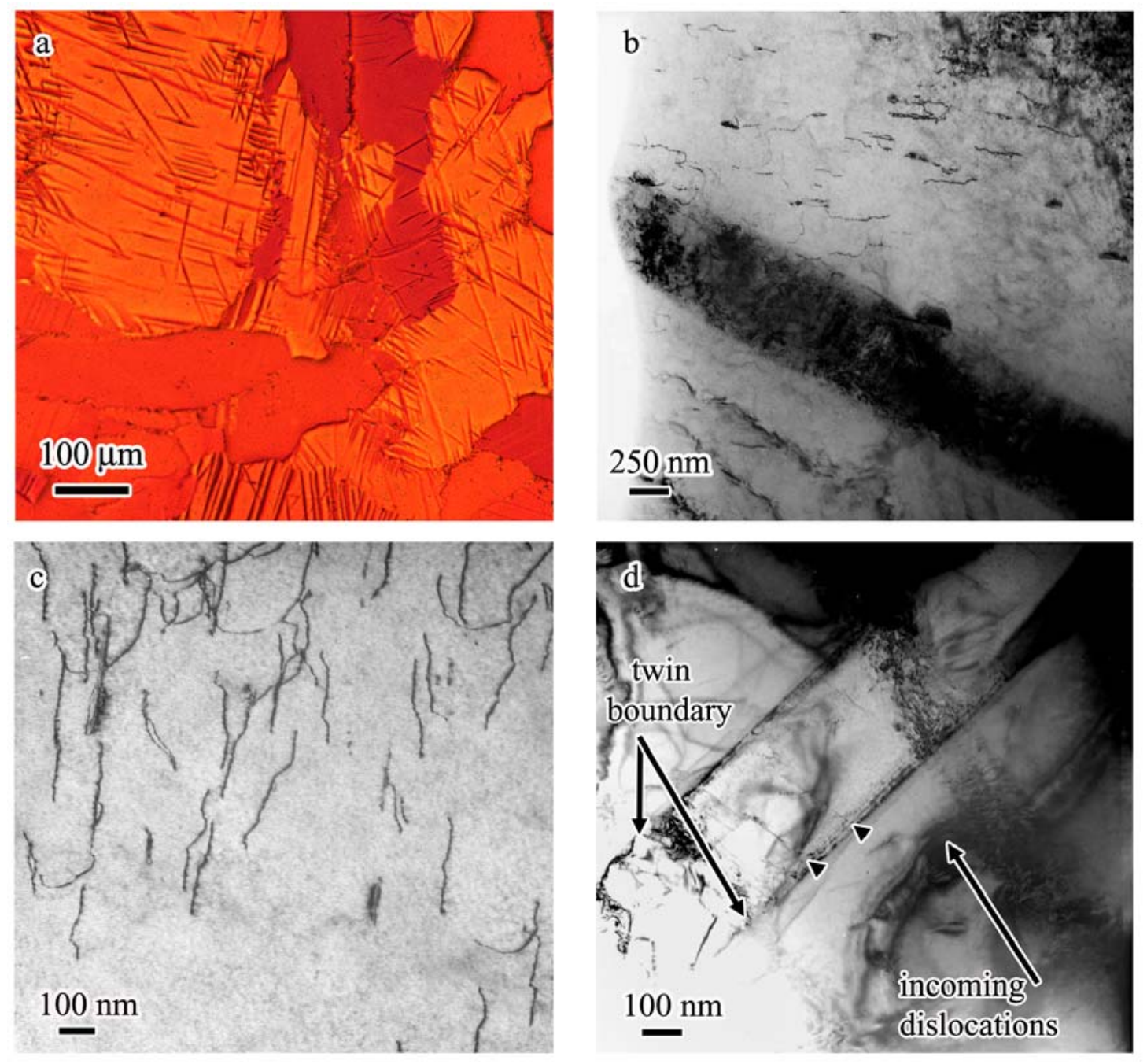

Figure 3 Deformed microstructure of zirconium bar. Dynamic compression was performed on this sample at a strain rate of $1200 / \mathrm{s}$ to a strain of $9 \%$. a) Optical micrograph showing twins in some grains. b, $c$ and d) bright-field TEM images showing twins and dislocations. 


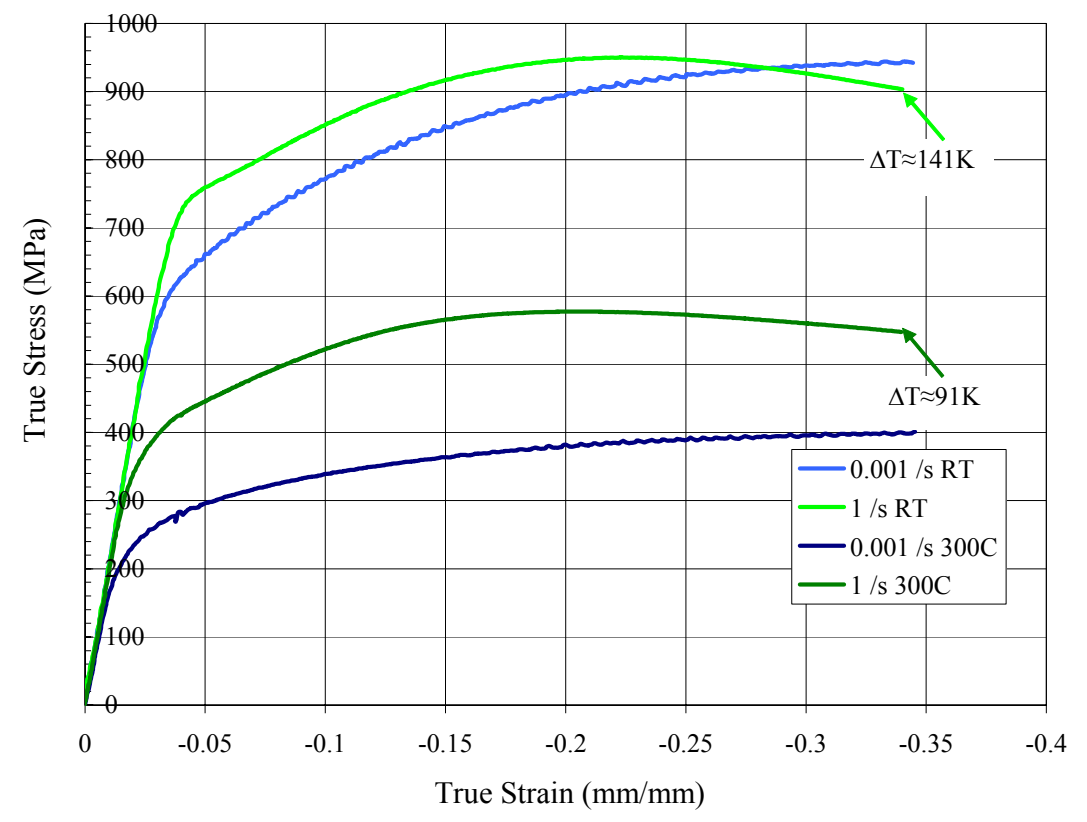

Figure 4 Quasi-static compressive stress-strain data for zirconium plate.

The characterization of the mechanical behavior of the zirconium bar and zirconium plate materials has been carried out, though not yet completed, for both quasi-static and dynamic conditions. Figure 4 shows the stress-strain response for the zirconium plate under quasi-static loading conditions, while Figure 5 shows the response for both the bar and plate materials under dynamic conditions. The dynamic stress-strain response of the zirconium bar material shows a slightly lower yield than the plate materials, possibly due to the larger grain size. The smoother overall appearance of the stress-strain curves of the bar material compared to the plate material is due to the fact that pulse shapers were used in these tests. The quasi-static and dynamic compression test data for the plate material in which saturation was observed were used to construct plots of the kinetics of yield strength and saturation stress. Figures

a

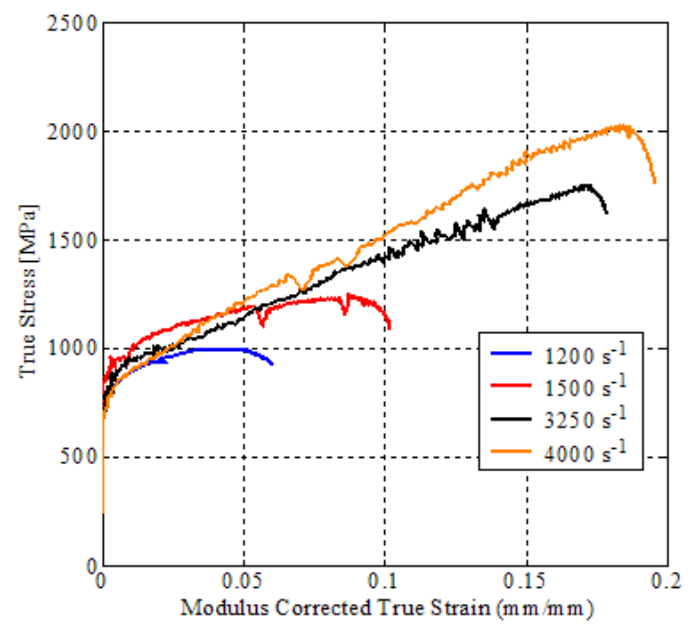

$\mathrm{b}$

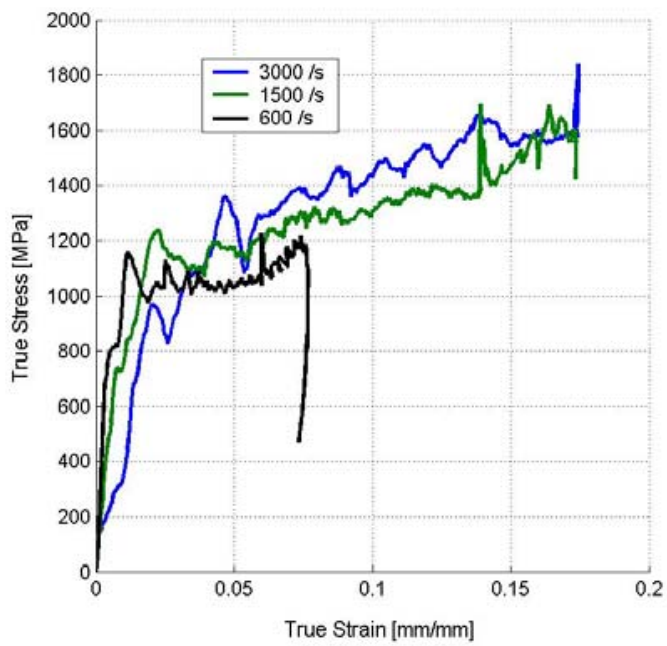

Figure 5 Dynamic stress-strain curves for (a) zirconium bar and (b) zirconium plate. 

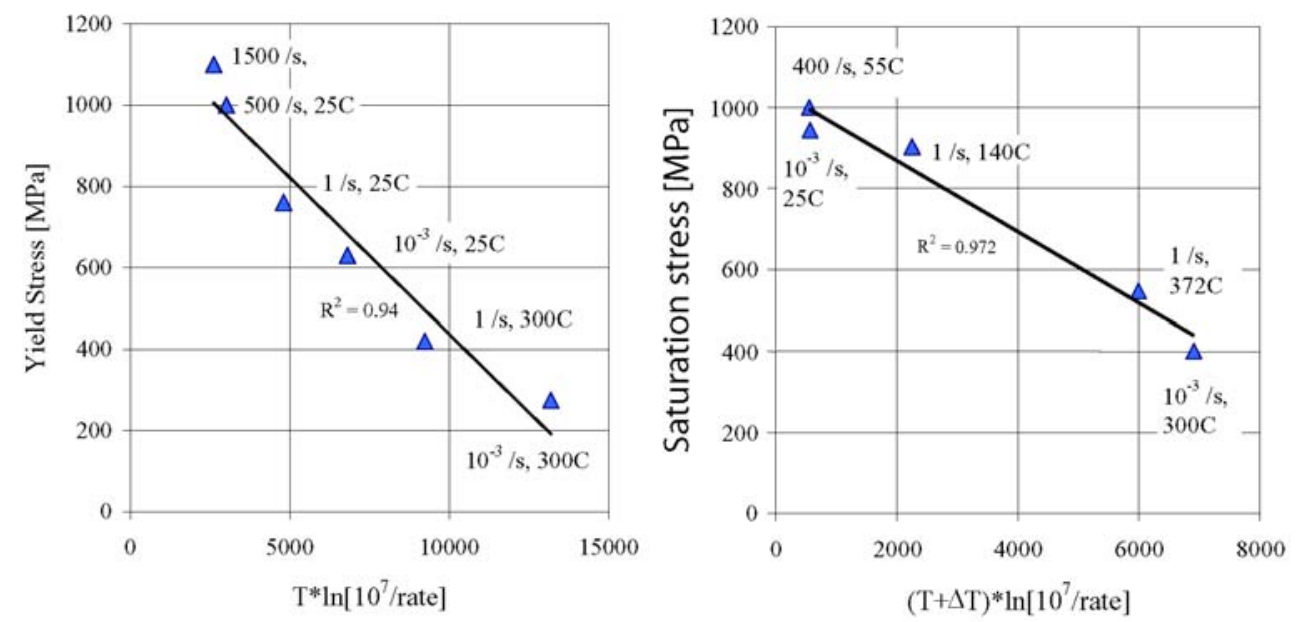

Figure 6 (a) Kinetics yield plot for zirconium plate. (b) Kinetics saturation stress plot for zirconium plate-including adiabatic temperature rise.

$6 \mathrm{a}$ and $6 \mathrm{~b}$ respectively show plots of yield and saturation stress versus temperature compensated strain rates. The resulting fits are linear. From Fig. 4, we can see that there is a significant softening behavior in the data for compression at a rate of $1 \mathrm{~s}^{-1}$. If we assume adiabatic test conditions for this rate, we can calculate an upper bound for the temperature rise due to the plastic work done on the sample by assuming $100 \%$ of the work goes into thermal energy. The saturation kinetics plot takes into account the calculated temperature rise as such.

The presence of twins in the bar material due to the deformation has already been demonstrated via optical microscopy (Fig. 3). Deformation twinning in the plate material is fully expected due to the basal orientation with respect to the stress axis, although the microstructure is currently being analyzed to confirm this hypothesis.

The evolution of crystallographic texture for both the bar and plate material has been examined using x-ray diffraction. In Fig. 7, we have confirmation that the deformed textures of the two materials have vastly different character. The plate material still has an oval shape to it, though it is rotated by approximately $90^{\circ}$ due to sample orientation in the goniometer, but the two peaks have been replaced by a single peak at a somewhat larger angle to the compression axis.
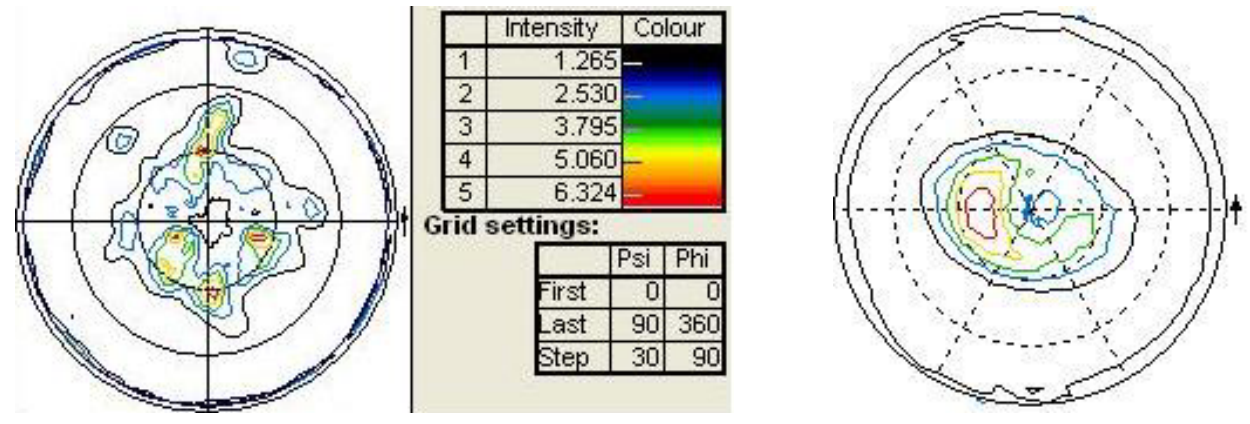

Figure 7 (a) Texture of deformed zirconium bar-4000/s, 18\%. (b) Texture of deformed zirconium plate- $3000 / \mathrm{s}, \mathbf{1 8 \%}$.

A new capability similar to a plate impact test but based on a pulsed laser loading technique has been developed, calibrated and initial tests have been performed. This new capability extends our strain rate test range into the range from $10^{4}$ to $10^{6} \mathrm{~s}^{-1}$ and introduces the capability of keeping the total strain 


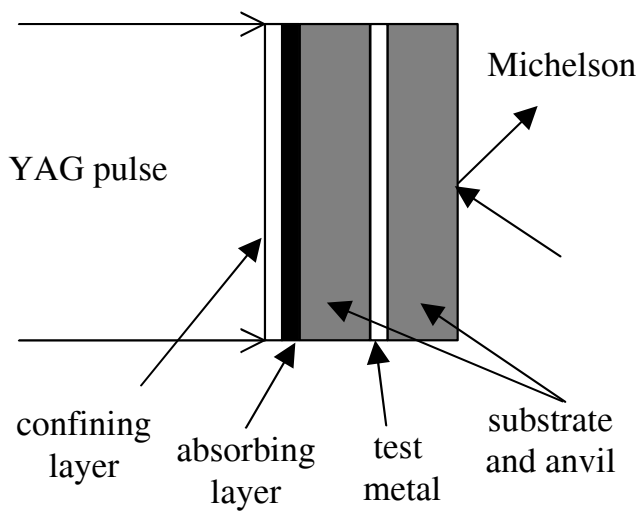

Figure 8. Laser loading set-up.

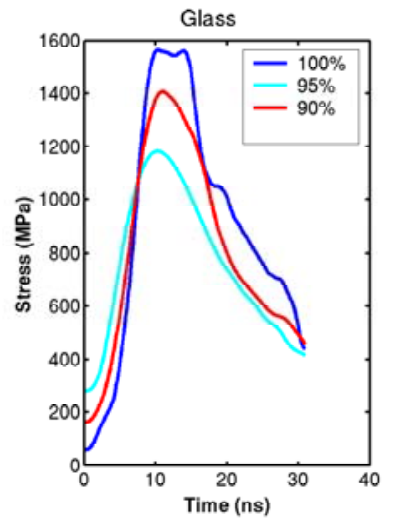

Figure 9: Input stress for various fleunces in a laser loading experiment.

small, and performing tests as a function of temperature. The setup is also compact. Figure 8 shows a schematic of the experimental set-up.

A pulsed infrared Nd:YAG laser (power $1 \mathrm{~J}$, duration $10 \mathrm{~ns}$, wavelength $1064 \mathrm{~nm}$ ) is focused to a $3 \mathrm{~mm}$ diameter using an objective lens, and is incident upon the surface of a silicon or fused quartz plate. The incident plate surface is coated with a thin (about $0.5 \mu \mathrm{m}$ ) energy-absorbing layer, usually aluminum. This layer absorbs the laser radiation in a short time period and consequently undergoes a rapid volumetric expansion. The expansion is constrained on one side by a $30 \mu \mathrm{m}$ confining layer that is transparent to the laser wavelength. Consequently, a severe and sudden compressive loading pulse propagates in the incident plate, with a rise time dependent on the material, thickness and rise time of the laser pulse ${ }^{2}$. The testing material, a disk of diameter $3 \mathrm{~mm}$ and thickness $200 \mu \mathrm{m}$, is sandwiched between the incident and anvil plates, which are made of the same material. Thus, the laser-induced compression wave loads the sample and is partially transmitted into the anvil plate. In a fashion it is very similar to plate impact, the transmitted compressive pulse travels to the free surface of the anvil where it is recorded by a Michelson interferometer as a surface displacement. This data can then be converted into surface velocity and eventually stress in the material using simple one-dimensional wave propagation arguments, which are valid until the time that reflections from the lateral boundaries reach the measurement point. Figure 9 shows typical input stress histories as a function of time for different values of laser fluence (= energy/area).

With the successful completion of this capability we have started to determine the deformation response of copper. Figures 10a and 10b show the incident and transmitted signals measured in shock loading experiments on single crystal and polycrystalline $\mathrm{Cu}$. The output signal expected if the $\mathrm{Cu}$ were to remain elastic is shown for comparison. The discrepancy between the elastic and the actual signal suggests significant plastic deformation has occurred. To verify this loading pulse had caused the sample to deform, the microstructure was examined using transmission electron microscopy. The TEM images shown in Figs. $10 \mathrm{c}$-e show the initial microstructure of the undeformed material in the single crystal $\mathrm{Cu}$, Fig.10c and polycrystalline copper, Fig. 10d; and the deformed microstructure in single crystal $\mathrm{Cu}$, Fig.10e and the polycrystalline material, Fig.10f. The peak input stress is $\sim 1.8 \mathrm{GPa}$ and the peak output stress is $\sim 650 \mathrm{MPa}$ for both copper samples. The microstructure is notable in the difference from the undeformed material in that both deformed materials show evidence of deformation twinning whereas no twins were observed in the undeformed material. In the single crystal, three deformation twins are seen crossing a series of bend contours. No dislocations are seen in this region which is surprising given the initial dislocation density, Fig. 10c. This difference is attributed to variation in the microstructure and not to a loss of dislocations during the deformation. Deformation twins were also created in polycrystalline material, Fig. 10f and Fig. 11a. In these examples the deformation twin has propagated through the preexisting dislocation structure, cutting the dislocations. The cut dislocations now terminate on the twin 
a

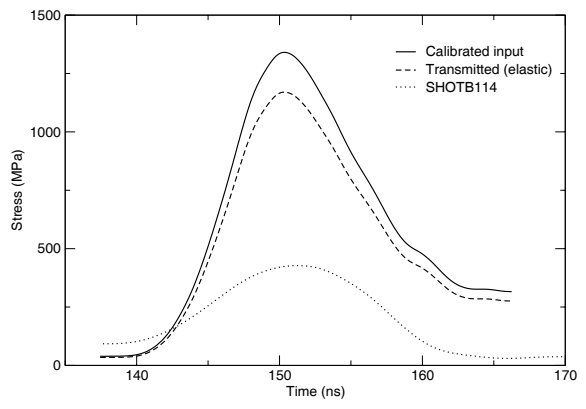

b

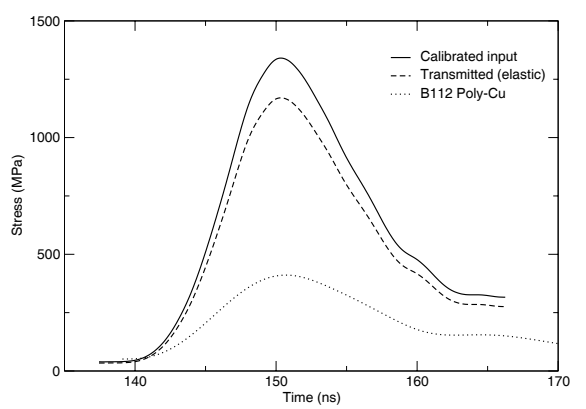

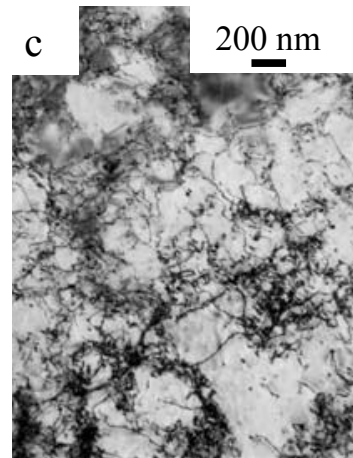
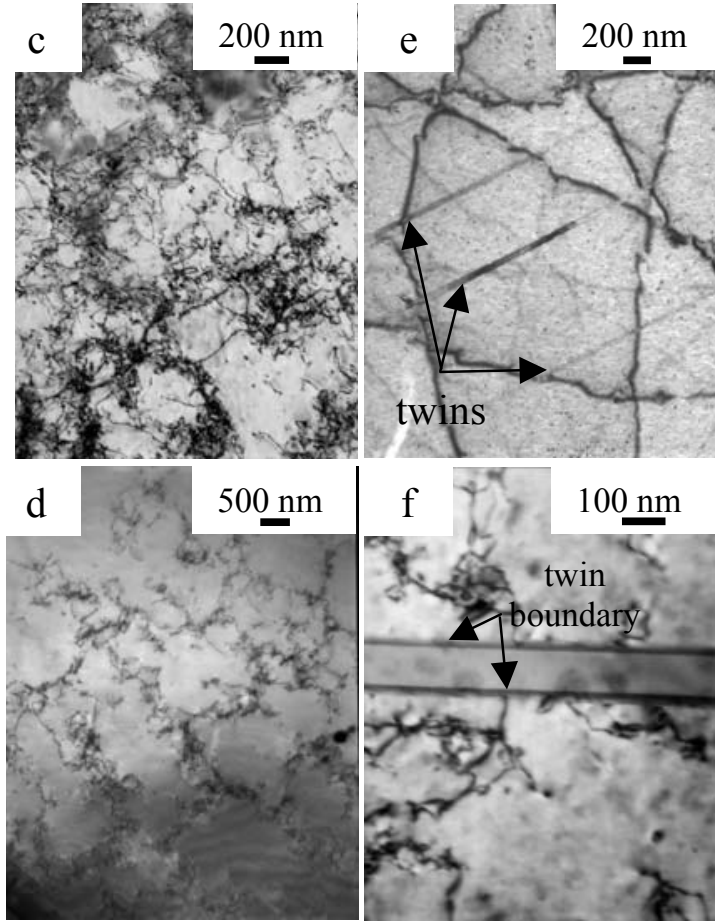

Figure 10: (a) Incident and transmitted stress in a shock loaded $\mathrm{Cu}$ single crystal at $10^{6} / \mathrm{s}$. b) Expected elastic transmitted stress also shown, c) bright-field image showing initial structure in the single crystal d) bright-field image showing initial structure in the polycrystal, e) twins in deformed single crystal and f)

boundary. Examination at higher magnification shows the presence of a nanotwin but few dislocations inside the larger twin; the nanotwin is indicated by the arrow. These deformation structures confirm that the sample experienced a stress high enough to create deformation twins and provides a mechanism to investigate the interaction of deformation twins with dislocation structures. The ability to control the sample temperature provides a means to control the deformation response to investigate the transition from slip to twin dominated behavior. A key feature is that the total strain in the laser loaded samples is low providing the opportunity to understand the details of the interactions. Similar interactions of twins with dislocation cell structures were observed in copper tested in compression in the SHPB, although the density of twins was higher in the laser-loaded sample.

One of the key parameters to be determined for grain boundaries is the dependence of the grain boundary strength, the slip transmissibility factor, as a function of grain boundary misorientation. The effectiveness of different grain boundaries on strengthening a material is apparent in the form of engineered grain boundary materials and in the more recent work on using deformation twins with a nanometer separation distance between them to strengthen the material. To understand this dependence
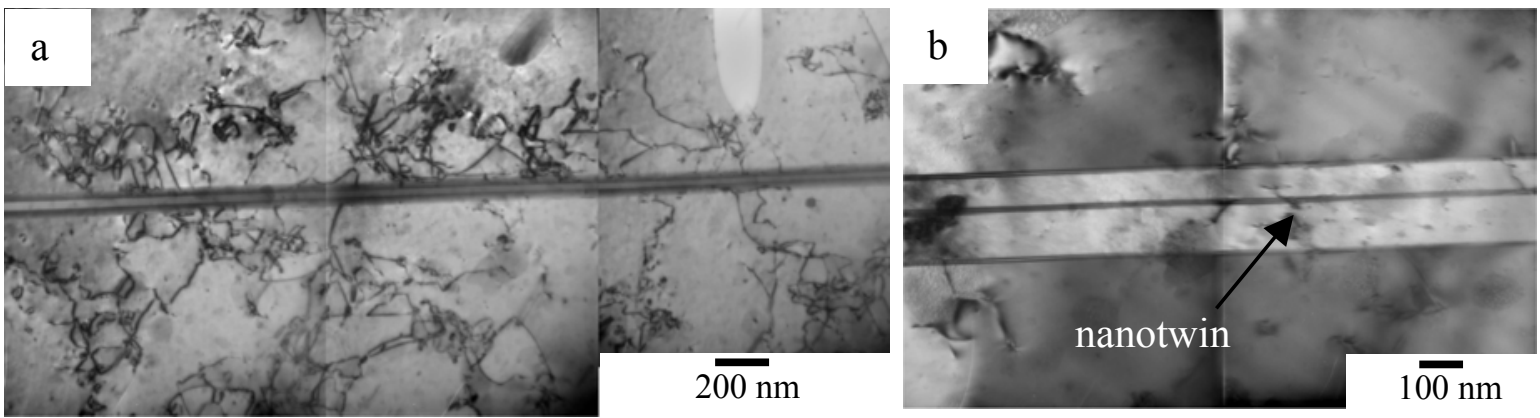

Figure 11: (a) Twin formation in polycrystalline $\mathrm{Cu}$, with successive sub-level nanoscale twinning. 
Table 3. Hardness measurements for grain boundaries in copper.

\begin{tabular}{|l|c|c|c|c|}
\hline \multicolumn{1}{|c|}{ material } & $\begin{array}{l}\text { Grain 1 } \\
\mathrm{GPa}\end{array}$ & $\begin{array}{l}\text { Boundary } \\
\mathrm{GPa}\end{array}$ & $\begin{array}{l}\text { Grain 2 } \\
\mathrm{GPa}\end{array}$ & boundary \\
\hline as-received OFHC copper & 2.22 & 3.77 & 3.02 & Unknown \\
\hline OFHC copper. annealed 700 ${ }^{\circ} \mathrm{C}$ for 7 hours & 2.03 & 2.12 & 2.0 & Unknown \\
\hline $\begin{array}{l}\text { Single crystal, tested in unaxial tension and then rolled to } \\
75 \% \text { reduction (No Recovery) }\end{array}$ & & 2.46 & & twin \\
\hline $\begin{array}{l}\text { Single crystal, tested in unaxial tension and then rolled to } \\
75 \% \text { reduction (Partial Recovery) }\end{array}$ & & 2.28 & & Twin \\
\hline $\begin{array}{l}\text { Engineered grain boundary distribution 65\% special } \\
\text { boundaries (supplied by LLNL) }\end{array}$ & 2.24 & 2.31 & 2.28 & Twin \\
\hline $\begin{array}{l}\text { Engineered grain boundary distribution 65\% special } \\
\text { boundaries (supplied by LLNL) }\end{array}$ & 2.28 & 2.35 & 2.27 & twin \\
\hline
\end{tabular}

and to give an "effectiveness" to the grain boundary it is necessary to measure the transmission factor as a function of misorientation. One approach to achieving this goal is to use nanoindentation to measure the hardness of the grain boundary or the region vicinal to it in conjunction with active straining experiments in situ in the transmission electron microscope.

Nano-indentation tests were conducted on copper subjected to various thermo-mechanical treatments using the following method. First, the samples were polished and lightly etched using standard metallographic techniques. Light etching yielded good contrast between the boundaries and the grains. Cross section scans were conducted prior to indention to verify that the surface in the vicinity of the boundary was relatively flat. Next, the samples were tested using a Hysitron nano-indention system. In a typical test, the boundary was subjected to a maximum load of $500 \mu \mathrm{N}$ applied at a rate of $100 \mu \mathrm{N} / \mathrm{s}$ in a trapezoidal loading profile (load for five seconds, hold for five seconds and unload). The values of hardness and elastic modulus were extrapolated from the unloading portion of the curve by using the system software. STM images of indented areas and the corresponding hardness measurements for a random grain boundary of unknown character in polycrystalline OFHC copper and for a twin grain boundary in $\mathrm{Cu}$ with an engineered grain boundary structure are presented in Fig. 12. The hardness values from different grain boundaries in copper subjected to different thermo-mechanical treatments are summarized in Table 3.

The hardness values of the different types of boundaries reported above tend to lie in a range

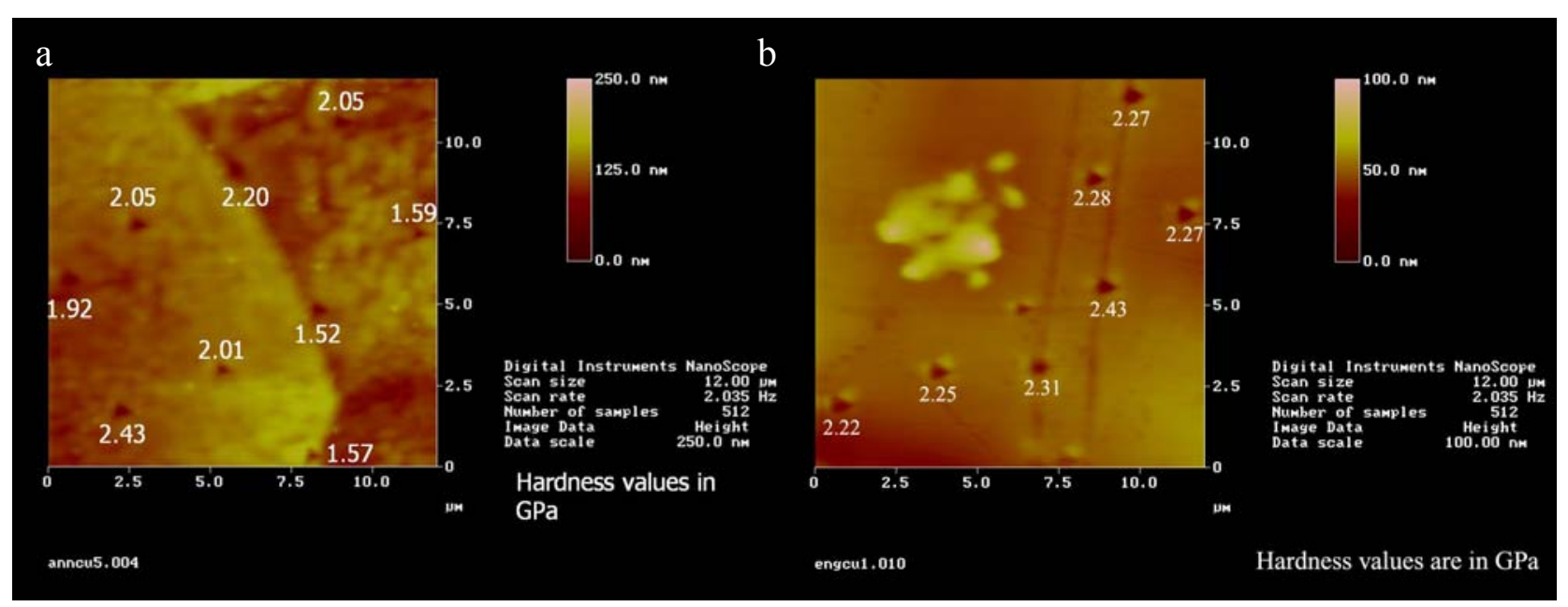

Figure 12. a) Hardness values on a random grain boundary in polycrystalline OFHC copper following an anneal at $450^{\circ} \mathrm{C}$ for 24 hours. b) Hardness measurements on a twin boundary in copper with engineered grain boundaries. 
from 2.1 to $2.5 \mathrm{GPa}$. One exception was for grain boundaries in as-received polycrystalline copper, which had a hardness of $3.77 \mathrm{GPa}$. This high value may reflect the underlying microstructure that was composed of a high dislocation density. Comparing these data and trying to determine whether boundary orientation is related to boundary strength is difficult because of the narrow range in the measured hardness values between the twin boundaries produced in the single crystal copper and the higher angle boundaries in the annealed polycrystal copper. Thus, making a statement as to the transmissibility of twin boundaries and higher angle grain boundaries cannot be made at this time.

To obtain better insight to the slip transmissibility of grain boundaries we propose to use the Focused Ion Beam to machine out columns with the grain boundary contained within the column. The indentation will then be applied parallel to the grain boundary plane normal, generating a deformation reaction that will spread to the grain boundary and either be transmitted through or blocked by the grain boundary. This sample geometry permits sectioning of the column to extract a TEM sample containing the grain boundary. The nature of the deformation, the extent of the transmission, and the reaction process will then be fully characterized and correlated with the hardness value. Secondly, deformation experiments will be performed in situ in the TEM to determine the nature of the interactions of dislocations and grain boundaries as a function of misorientation.

\section{Modeling.}

One of the challenges for plasticity codes, especially as progress is made toward a multiscale approach, is the transition from single to polycrystalline materials especially in the elasto-plastic transition region. Early work by Robertson and coworkers ${ }^{3-7}$ established experimentally the conditions for slip transfer across high-angle and random grain boundaries during the early stages of quasi-static loading. Specifically, it was determined that the slip transfer process was governed by competition between the slip system experiencing the maximum local resolved shear stress (applied stress + dislocation pile-up contribution) and the change in energy of the grain boundary as a consequence of the dislocation absorption and emission process. More recently these conditions have been shown applicable to interfaces between different materials or phases. ${ }^{8-11}$ Hoagland et al. ${ }^{12}$ have demonstrated that the stress transmission through a semi-coherent (or nearly incoherent) bi-material boundary is affected by the core structure of misfit dislocations in the boundary. Spreading of the core can lead to a boundary that has increased mobility of misfit dislocations and concomitant weakening of the boundary in shear. Hence, one must consider boundary structure on slip transfer. This is a complex problem as the boundary structure is not necessarily constant along the length of the grain boundary, creating weak and strong regions.

The challenge then is to formulate these slip transmission rules, at least in a phenomenological sense, into models that can be included in polycrystalline plasticity codes. One of the major achievements this fiscal period has been the development of appropriate models to account for slip transfer across interfaces. Basically, this involves accounting for the grain boundary by considering a bicrystal inclusion as the representative volume element of the aggregate. Each component of the bicrystal is subjected to a viscoplastic constitutive law and the response of the inclusion is determined based on the volume fraction of the components. Compatibility and equilibrium requirements are imposed across the interface. Interaction between the inclusions and the aggregate is accounted for through a visco-plastic selfconsistent scheme. The grain boundary misorientation is accounted for by the bicrystal and the local stress distribution through the viscoplastic constitutive law. To account for the change in the grain boundary energy, we propose:

- development of a "latent hardening" applied to slip systems which intersect the boundary;

- using an effective "back stress" created as a result of the residual dislocation produced as the difference between the absorbed and emitted dislocations. 
The first approach applies to boundaries with characteristics of high mobility of misfit dislocations such as at a bi-material interface. In collaboration with Los Alamos National Laboratory, we have applied this concept to account for the texture development in $\mathrm{Cu} / \mathrm{Nb}$ multilayers. This work has been accepted for publication in Phil. Mag ${ }^{13}$. The latter approach is applicable to slip transmission across grain boundaries, including twin boundaries. In the following, we briefly comment on our on-going experimental study of the effect of twin boundaries on deformation and consider modeling the stress strain curve of silver that had been cold-rolled and then annealed to produce annealing twins within grains with an average diameter of $2 \mu \mathrm{m}^{14}$. The resulting stress-strain curve is shown in Fig. 13a. The tensile properties are characterized by a relatively low proportional limit (on the order of $20 \mathrm{MPa}$ ), followed by an elongated yield point at $140 \mathrm{MPa}$, and finally the usual stage III hardening behavior beyond a strain of a few percent. The challenge is to understand the evolution of plasticity in this material. To understand the microstructural evolution with strain some tensile tests were interrupted at strains of 0.001 and $0.01 \%$ and the microstructure assessed by TEM. Additional information can be found in ref. ${ }^{15}$

Following the anneal and prior to quasi-static testing at room temperature the microstructure was
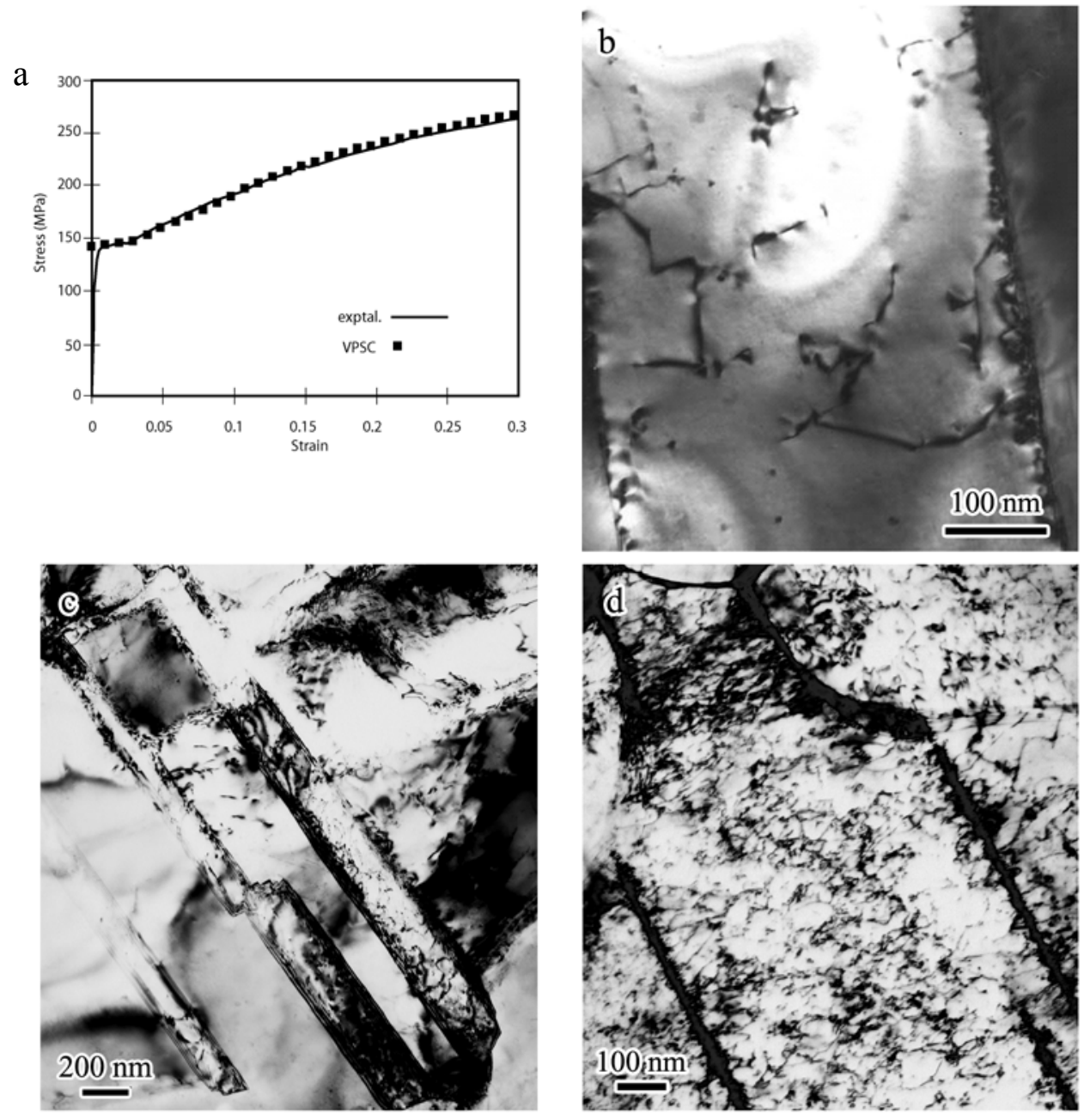

Figure 13. a). stress strain curve of fine grained material, experimental and model; b). initial microstructure; c). microstructure at strain of $0.001 \%$ and d). microstructure at strain of $0.01 \%$. 
dominated by annealing twins within the $2 \mu \mathrm{m}$ grains. These twins contained a high degree of disorder as shown in Fig. 13b. Dislocation half-loops are seen emerging from the twin boundaries, and weak-beam dark-field images (not shown) reveal a high density of dislocations within the boundaries. The dislocation density elsewhere in the specimen is low. Following testing, the evolution of the microstructure to the apparent yield point is shown in Fig. $13 \mathrm{c}(\mathrm{e}=0.001 \%)$ and $13 \mathrm{~d}(\mathrm{e}=0.01 \%)$. High densities of dislocations now exist within and outside the annealing twins and some twin boundaries appear to have been penetrated by dislocations, Fig 13d. These observations imply that the annealing twin boundaries were sources of partial and perfect dislocations; a similar suggestion has been made by others. $^{16,17}$ To validate this suggestion, in situ straining experiments were performed using preloaded specimens. This study showed that perfect and partial dislocations are emitted from the annealing twins, that the annealing twins can act as barriers to slip and in other cases slip is transmitted across the twin boundary, that deformation twins are produced and annihilated during the straining process, and, lastly, that annealing twins can be destroyed through the interaction with perfect and partial dislocations. This provides us with a picture of the microstructure evolution as a function of strain. The challenge was to capture the key elements in the model.

To model the dislocation source generation from the annealing twin boundaries it is necessary to consider the evolution of both mobile, $\rho_{\mathrm{m}}$, and forest, $\rho_{\text {for }}$, dislocation densities during deformation. That is, two internal variables are used; a similar approach has been used to study deformation in metals that involves rapid changes of deformation path. ${ }^{18,19}$ Following Kubin and Estrin, ${ }^{20}$ the evolution of the mobile and forest dislocation densities can be expressed as:

$$
\begin{gathered}
\dot{\rho}_{m}=\left(k_{1}-k_{2} \rho_{m}-k_{3} \sqrt{\rho_{f}}\right) \sum_{\alpha}\left|\dot{\gamma}^{\alpha}\right| \\
\text { and } \dot{\rho}_{f}=\left(k_{2} \rho_{m}+k_{3} \sqrt{\rho_{f}}-k_{4} \rho_{f}\right) \sum_{\alpha}\left|\dot{\gamma}^{\alpha}\right|
\end{gathered}
$$

In these equations, $\mathrm{k}_{1}, \mathrm{k}_{2}, \mathrm{k}_{3}$ and $\mathrm{k}_{4}$ represent the generation of mobile dislocations, the annihilation and trapping of mobile dislocations, the immobilization of dislocations as they interact with forest dislocations, and dynamic recovery, respectively.

These equations are introduced into a composite grain model in which each composite consists of two phases separated by a $\Sigma 3$ twin boundary. Each composite grain is treated as a viscoplastic ellipsoid embedded in a Homogeneous Equivalent Medium, HEM. Both individual grains and HEM have uniform properties, where the latter represents the aggregate by averaging the response of all composite grains. The viscoplastic self-consistent scheme of Lebensohn and Tomé ${ }^{21}$ is used to solve for the heterogeneous plasticity response for given boundary conditions. The macroscopic response is characterized by small dislocation trapping $k_{2}$ and immobility $k_{3}$ coefficients, and large generation $k_{1}$ and annihilation $k_{4}$ coefficients. The results of the model are shown in Fig 13a. The essential feature of the macroscopic response, the elongation of the yield point, is captured by the model.

This work was essential complete in the previous fiscal period but the issue of decreasing the importance of the annealing twin boundaries as sources and as obstacles for dislocations as a function of strain could not be justified physically. This period we have shown that the annealing twins are destroyed by the interaction with both perfect and partial dislocations. An example of this destruction can be seen in Fig. 13d in which the twin boundary is clearly separated and in Fig 14. The micrographs and schematic in Fig. 14 show the interaction of partial dislocations with an annealing twin boundary that results in the transmission of slip across the twin boundary and the subsequent destruction of the boundary. This destruction can be understood in terms of the interaction between the incoming and outgoing dislocations and the twin boundary. The dislocation created in the twin boundary as a consequence of the dislocation absorption and emission is mobile in the boundary and the motion of it initiates the detwinning reaction. These observations provide a physical basis for diminishing the importance of the annealing twins as sources and as barriers to deformation as a function of strain. This work was presented in the Dislocation 

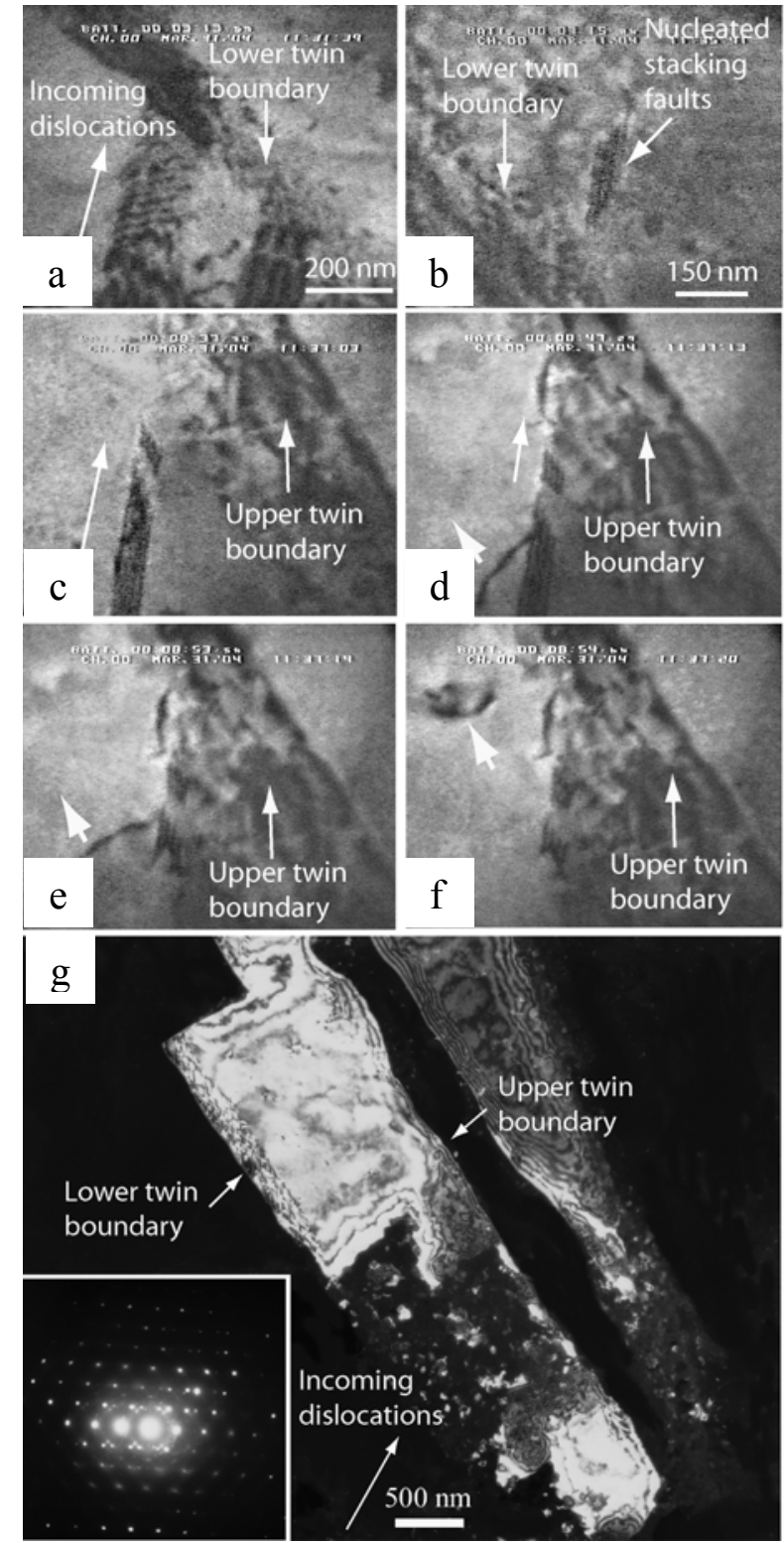

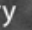

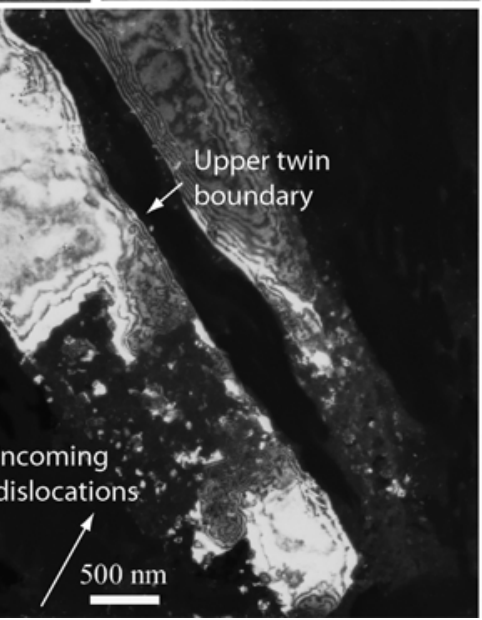

Figure 14 Interaction of partial dislocations with an annealing twin boundary as a function of strain. A) interaction of partial dislocations with lower twin boundary, b) slip transmission, c) movement of partial dislocations across twin boundary, $d-f$ ) interaction of partial dislocations with upper twin boundary, g) final configuration showing annihilation of a portion of the twin, h) schematic showing initial interaction (corresponds to a), i) schematic showing transmission, corresponding to $b$ and $j$ ) final configuration corresponding to $\mathrm{g}$.

2004 meeting and a short paper has been accepted for publication in Materials Science and Engineering ${ }^{15}$. A detailed manuscript is being prepared for submission to an archival journal.

Current effort is directed at a parallel implementation of the Field Dislocation Mechanics framework, posed by Acharya ${ }^{22-25}$. Along with parallel codes for the incompatibility and equilibrium problems, an explicit (Galerkin-Least Squares) technique has been developed for the transport of excess dislocation density. Verification efforts are underway. While it is not our specific intention to model individual dislocations, but rather operate at a mesoscale representing dislocation arrays, single dislocation examples provide welcome examples for verification. We have computed stress fields for 
individual dislocations, as well as tilt and twist boundaries, and demonstrated agreement with analytical solutions. As an example of capability to simulate transport of dislocation density, results for the operation of a Frank-Read source are shown in Figure 15. Note that no special action is necessary to handle the annihilation of opposite edge segments, as shown in State 26, this annihilation being inherent in the transport equation.

This effort has led to contact with the Hamilton-Jacobi equations, classically associated with geometrical optics. This significance in the present context lies in the capability to model the development and motion of a front using our explicit code. We are at present exploiting this capability to simulate the motion of a twinning front, expressed as a continuous distribution of partial dislocation density. Following Acharya, we are pursuing the coupling of FDM with crystal plasticity models. It is then our objective to model concurrent slip and twinning, with description for the growth of twins by FDM.

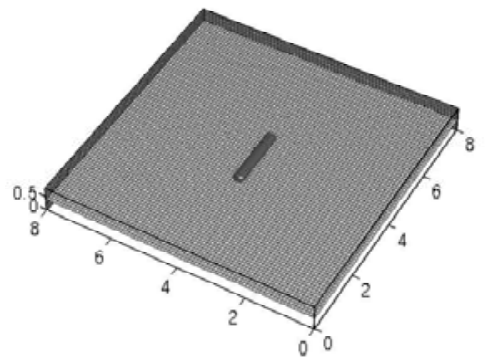

Initial Condition

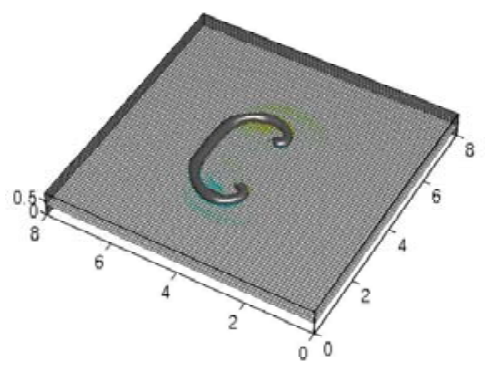

State 14

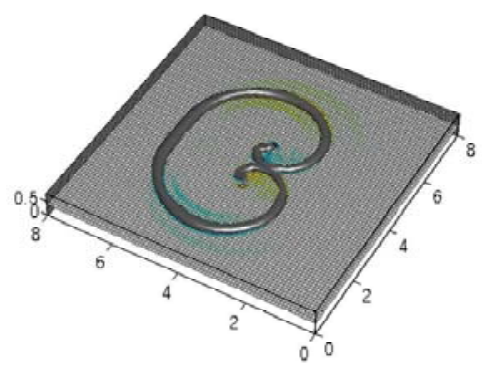

State 24

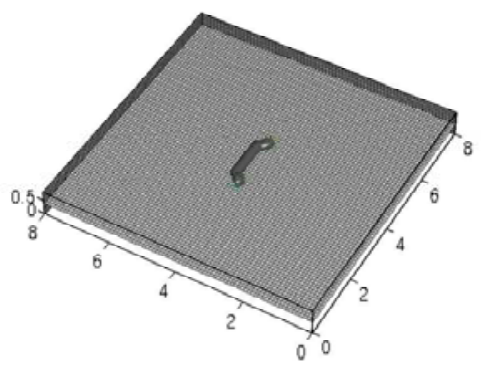

State 2

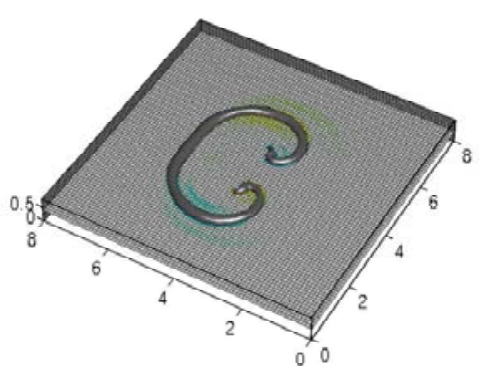

State 19

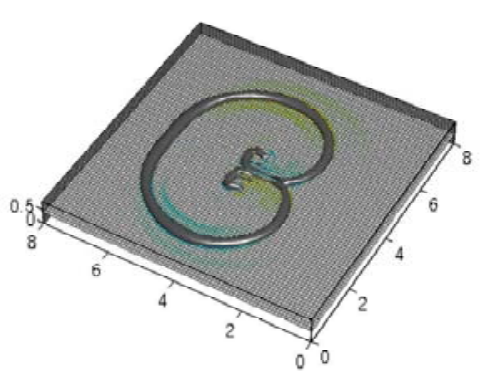

State 26

Figure 15 . Continuum model of a Frank-Read source. Grey-shaded surface represents a threshold level of $40 \%$ peak value of the initial screw segment. The blue/yellow shading give indicate of negative/positive edge density of a (numerical) wake. 


\section{References}

1. Y. T. Zhu, X. Z. Liao, S. G. Srinivasan, Y. H. Zhao, M. I. Baskes, F. Zhou and E. J. Lavernia:Nucleation and growth of deformation twins in nanocrystalline aluminum, Applied Physics Letters, 85 (21), 5049 (2004).

2. J. Wang, R. L. Weaver and N. R. Sottos:A parametric study of laser induced thin film spallation, Experimental Mechanics, 42 (1), 74 (2002).

3. W. A. T. Clark, R. H. Wagoner, Z. Y. Shen, T. C. Lee, I. M. Robertson and H. K. Birnbaum:On the criteria for slip transmission across interfaces in polycrystals, Scripta Metallurgica et Materialia, 26 (2), 203 (1992).

4. T. C. Lee, I. M. Robertson and H. K. Birnbaum:TEM in situ deformation study of the interaction of lattice dislocations with grain boundaries in metals, Philosophical Magazine A, 62 (1), 131 (1990).

5. T. C. Lee, I. M. Robertson and H. K. Birnbaum:An in situ transmission electron microscope deformation study of the slip transfer mechanisms in metals, Metallurgical Transactions A, 21A (9), 2437 (1990).

6. T. C. Lee, R. Subramanian, I. M. Robertson and H. K. Birnbaum:Dislocation-grain boundary interactions in $\mathrm{Ni}_{3} \mathrm{Al}$ effects of structure and chemistry, Scripta Metallurgica et Materialia, 25 (6), 1265 (1991).

7. J. Shirokoff, R. I. M. and B. H. K., in Defect - Interface Interactions Symposium., edited by., (Mater. Res. Soc., Pittsburgh, PA, USA.,City, 1994),pp 263.

8. A. Misra and R. Gibala:Plasticity in multiphase intermetallics, Intermetallics, 8 (9-11), 1025 (2000).

9. M. A. Gibson and C. T. Forwood:Slip transfer of deformation twins in duplex \&gamma;based Ti-Al alloys Part III. Transfer across general large-angle \&gamma;-\&gamma; grain boundaries, Philosophical Magazine A, 82 (7), 1381 (2002).

10. J. M. K. Wiezorek, X.-D. Zhang and H. L. Fraser, in Symposium A, Multiscale Phenomena in Materials-Experiments and Modeling, Nov 30-Dec 2 1999, edited by., (Materials Research Society, Warrendale, PA, USA,City, 578, 2000), pp 383.

11. Y. G. Zhang, F. D. Tichelaar, F. W. Schapink and M. C. Chaturvedi:Interaction of deformation twins with $\alpha_{2}$-plates in $\gamma$-TiAl base alloy deformed at room temperature, Materials Science \& Engineering A, A219 (1-2), 162 (1996).

12. R. G. Hoagland, R. J. Kurtz and C. H. Henager Jr.:Slip resistance of interfaces and the strength of metallic multilayer composites, Scripta Materialia, 775 (2004).

13. K. AL-Fadhalah, C. N. Tomé, A. J. Beaudoin, I. M. Robertson, J. P. Hirth and A. Misra $^{3}$ :Modeling Texture Evolution During Rolling of $\mathrm{Cu}-\mathrm{Nb}$ Multilayered System, Philosophical Magazine, in press (2005).

14. D. A. Korzekwa, Los Alamos National Laboratory supplied the material in this condition.

15. I. M. Robertson, A. Beaudoin, K. Al-Fadhalah, C.-M. Li, J. Robach, B. D. Wirth, A. Arsenlis, D. Ahn and P. Sofronis:Dislocation-obstacle interactions: dynamic experiments to continuum modeling, Materials Science \& Engineering A, in press (2005).

16. J. E. Flinn, D. P. Field, G. E. Korth, T. M. Lillo and J. Macheret:The flow stress behavior of OFHC polycrystalline copper, Acta Materialia, 49 (11), 2065 (2001).

17. D. P. Field, B. W. True, T. M. Lillo and J. E. Flinn:Observation of twin boundary migration in copper during deformation, Materials Science and Engineering A, 372 (1-2), 173 (2004). 
18. Y. Estrin, in High Temperature Constitiutive Modelling - Theory and Application, Dec 1-6 1991, edited by., (Publ by ASME, New York, NY, USA,City, 26, 1991),p.^pp 65.

19. Y. Estrin, H. Braasch and Y. Brechet:Dislocation density based constitutive model for cyclic deformation, Journal of Engineering Materials and Technology, Transactions of the ASME, 118 (4), 441 (1996).

20. L. P. Kubin and Y. Estrin:Evolution of dislocation densities and the critical conditions for the Portevin-Le Chatelier effect, Acta Metallurgica, 38 (5), 697 (1990).

21. R. A. Lebensohn and C. N. Tomé: A selfconsistent approach for the simulation of plastic deformation and texture development of polycrystals: application to zirconium alloys, Acta Metall. Mater., 41, 2611-2624. (1993).

22. A. Acharya: A model of crystal plasticity based on the theory of continuously distributed dislocations, JMPS, 49, 2001, 761-784.

23. A. Acharya: Driving forces and boundary conditions in continuum dislocation mechanics, Proc R Soc Lond A, 459, 2003, 1343-1363.

24. A. Acharya: Constitutive analysis of finite deformation field dislocation mechanics, JMPS, 52, 2004, 301-316.

25. A. Roy, and A. Acharya: Finite element approximation of field dislocation mechanics, JMPS, 53, 2005, 143-170. 